\title{
Towards Teacher-managed Deployment and Integration of Non-SaaS Tools in Virtual Learning Environments
}

\author{
Rafael Cano-Parra, Eduardo Gómez-Sánchez, Miguel L. Bote-Lorenzo, and \\ José A. González-Martínez \\ GSIC/EMIC research group, Universidad de Valladolid, Valladolid, Spain \\ rafcano@gsic.uva.es, edugom@tel.uva.es, migbot@tel.uva.es, \\ jgonmar@gsic.uva.es
}

\begin{abstract}
Virtual Learning Environments (VLEs) such as Moodle or Sakai are now commonplace in many educational settings, but the limited set of built-in tools (e.g. chats or forums) is often mentioned as a drawback. While successful solutions to integrate Software as a Service (SaaS) tools are reported in the literature (e.g. IMS Basic LTI and GLUE), there are other non-SaaS tools with potential for education that cannot be integrated in VLEs. These include single-user standalone tools that are run on local computers without a web-based interface (e.g. Matlab), but also virtual machines and laboratories that are common in Computer Science-related disciplines. This paper addresses the integration of these non-SaaS tools in VLEs, proposing an architecture to help teachers to select and configure non-SaaS tools using existing integration approaches from VLEs, but that also relies on cloud infrastructures for provisioning and deploying these tools transparently to the end users.
\end{abstract}

Keywords: architecture · cloud computing · e-learning · educational tools · integration · software as a service · virtual learning environments

\section{Introduction}

Virtual Learning Environments (VLEs) typically allow educators to structure the learning activities in courses or lessons, describing for each activity a set of predefined learning objectives, and including some built-in tools and artifacts (e.g. chats, forums, or documents) to help students to reach these objectives [4]. Several authors, like Pätzold [7], argue that the support of learning activities with VLEs has benefits both for educators and students in some learning contexts. But one of the limitations in current VLEs, such as Moodle ${ }^{1}, \mathrm{LAMS}^{2}$, or Sakai ${ }^{3}$, is the lack of tools beyond the built-in tools [1,2]. Educators want to find in VLEs those tools that they are already using in their classes (e.g. Eclipse or Matlab) to implement learning situations using these tools inside of the VLE [9].

\footnotetext{
${ }^{1}$ https://moodle.org/

${ }^{2}$ http://lamsinternational.com/

${ }^{3}$ https://www.sakaiproject.org/
} 
The integration of Software as a Service (SaaS) tools in VLEs is now a reality thanks to some proposals such as IMS Basic LTI ${ }^{4}$ or GLUE! [1]. Under a predefined contract between the VLE and the SaaS tool, and including a small module in the VLE that allows to embed its web-based interface, these proposals can integrate instances of external tools while supporting educators in the management of their life-cycle (i.e. selection, configuration, and deletion).

Unfortunately, the integration of non-SaaS tools in VLEs is not supported by any of the existing proposals using an easy and generic way. However, there is a wide range of non-SaaS tools that are frequently used in various educational contexts, such as single-user standalone software commonly installed in personal computers (e.g. Matlab to do mathematical calculations), terminals of operating systems (e.g. a GNU/Linux terminal to compile and execute a program in C), and virtual laboratories (e.g. a virtual laboratory to conduct experiments on simulated networks using ns-3 simulator and Eclipse as a scripting environment).

There are several works in the literature related with the problem of integrating non-SaaS tools in VLEs. Mulfari et al. [6] propose an architecture for running assistive technology tools (software for disabled people) in a cloud and offering access to them through simple web browsers. Despotović-Zrakić et al. [3] propose ELABCloud, a system that allows the allocation and deployment of virtual machines associated to Moodle courses and users. Students can use preconfigured images that contain tools or platforms required by teachers, but this configuration must be done by specialized administration staff and out of the Moodle environment. Rădulescu's work [8] proposes the use of a middleware to fill the gap between Cloud Management Systems, employed in this study to facilitate the creation and management of virtual laboratories, and VLEs.

These works share two important limitations. First, both teachers and students need to go outside of the VLE to configure and interact with non-SaaS tools because there is no interface integration in the VLE. Even some non-SaaS tools require to work with third-party applications that have no access from a web browser. Second, teachers cannot manage the non-SaaS tool instance lifecycle as they can do with SaaS tools, so the selection, configuration, and deletion of one or more non-SaaS tool instances can be a complex and time-consuming task that cannot be handled by most educators.

Taking into account previous works and the relevance of using external tools inside of a VLE, we propose an architecture that enables the integration of nonSaaS tools in VLEs to help teachers to implement and deploy their current and new learning scenarios directly from the VLE, and using the cloud infrastructure to manage these tools.

\section{Architecture}

Consider a real scenario on which an educator wants his students to use Eclipse as a standalone tool to practice with Java language within the context of Moodle as a VLE that already employs GLUE! as a SaaS integration solution. He

\footnotetext{
${ }^{4}$ http://www.imsglobal.org/lti/
} 
wants that their students can collaboratively work with Eclipse in pairs, using a preconfigured environment which meets the teacher's requirements. There are no solutions that integrate Eclipse in a similar way as Google Docs, already used by students to write reports collaboratively. Also, the teacher could gain time and reduce complexity if the preconfigured environment can be automatically replicated for each students' group.

The architecture proposed in the Fig. 1 has been conceived to enable the integration of non-SaaS tools, such as Eclipse, either employing existing SaaS integration solutions, such as GLUE!, or developing ad-hoc adapters for VLEs. In this way, different integration options will be available for VLE administrators, so that it is more likely that they find one suitable for their needs. Furthermore, the architecture has been designed to enable the integration of non-SaaS tools deployed in cloud infrastructures. This allows the possibility of having a wide range of non-SaaS tools available for integration as well as of scaling the tools if it is necessary to cope with the load generated by students [5].

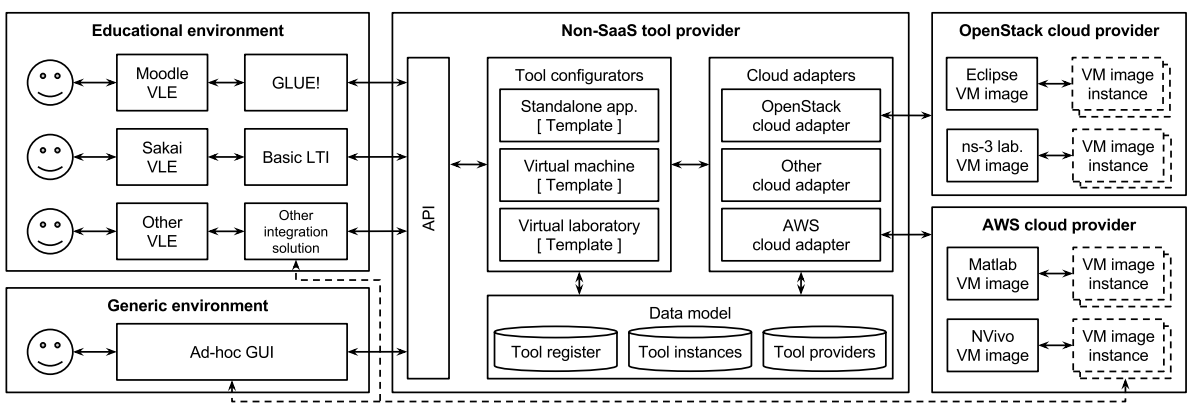

Fig. 1. The architecture that supports the integration of non-SaaS tools in VLEs.

Following the previous scenario, the educator can now choose to add an external tool in Moodle, which uses GLUE! to retrieve a list of non-SaaS tools available in the proposed architecture. The teacher selects Eclipse and fills a configuration template also provided by the proposed architecture that is stored in the data model databases. Once the Eclipse tool instance is configured, the architecture creates a cloud-independent script that is the translated by a cloud adapter into a sequence of commands that is required to launch the virtual machine(s), hosting the Eclipse tool in a specific cloud infrastructure (e.g. OpenStack) used by the tool provider. The virtual machine includes a visualization mode that translates the Eclipse interface to HTML5 and JavaScript using a web-socket proxy such as Guacamole or noVNC [6], so that the Eclipse interface can be seamlessly integrated in Moodle (or other VLE) course activity web page.

Other learning scenarios that require to use different non-SaaS tools, such as virtual machines or laboratories can be deployed and integrated in a similar way. The educator should fill out the template with his configuration needs 
(e.g. type of operating system, required services, network connection), and this information will be injected into the cloud adapters that will set up and launch the virtual infrastructure. Then, the console(s) interface(s) will be translated as a web-based content for their integration in the VLE, but also a simple access URI (e.g. IP address for SSH or VNC connection) can be passed, so that only the tool instance life-cycle management is used.

In summary, the proposed architecture will allow educators to manage themselves the configuration and integration in their VLEs of non-SaaS tools deployed in cloud infrastructures. This proposal will be prototyped in the short term using GLUE! middleware for integration in Moodle, OpenStack cloud infrastructure for deployment, and noVNC for serving standalone-tool web-based interfaces of standalone tools. Mid term efforts will extend this prototype, and will also be evaluated in diverse real scenarios.

Acknowledgements. This research has been partially funded by the European project 531262-LLP-2012-ES-KA3-KA3MP, the Regional project VA277U14, and the predoctoral fellowship FPI-UVa 2012.

\section{References}

1. Alario-Hoyos, C., Bote-Lorenzo, M.L., Gómez-Sánchez, E., Asensio-Pérez, J.I., Vega-Gorgojo, G., Ruiz-Calleja, A.: GLUE!: An architecture for the integration of external tools in Virtual Learning Environments. Computers \& Education 60(2013), 122-137 (Jan 2013)

2. Bower, M., Wittmann, M.: A Comparison of LAMS and Moodle as Learning Design Technologies - Teacher Education Students' Perspective. Teaching English with Technology - Special Issue on LAMS and Learning Design 11(1), 62-80 (2011)

3. Despotović-Zrakić, M., Simić, K., Labus, A., Milić, A., Jovanić, B.: Scaffolding Environment for e-Learning through Cloud Computing. Educational Technology \& Society 16(3), 301-314 (2013)

4. Dillenbourg, P., Schneider, D., Synteta, P.: Virtual Learning Environments. In: Proc. $3^{\text {rd }}$ Hellenic Conference with International Participation "Information \& Communication Technologies in Education" (EICT 2002). pp. 3-18. Rhodes, Greece (Sep 2002)

5. González-Martínez, J.A., Bote-Lorenzo, M.L., Gómez-Sánchez, E., Cano-Parra, R.: Cloud computing and education: A state-of-the-art survey. Computers \& Education 80(2015), 132-151 (Jan 2015)

6. Mulfari, D., Celesti, A., Villari, M., Puliafito, A.: Using Virtualization and noVNC to Support Assistive Technology in Cloud Computing. In: Proc. 2014 IEEE $3^{\text {rd }}$ Symposium on Network Cloud Computing and Applications (NCCA 2014). pp. 125-132. Rome, Italy (Feb 2014)

7. Pätzold, H.: Increasing value without increasing effort? The use of WebCT in accompanying face-to-face lectures under the constraint of low budget. International Journal of E-Learing and Distance Education 20(2), 78-84 (2005)

8. Rădulescu, S.A.: A perspective on E-learning and Cloud Computing. Procedia Social and Behaviour Sciences 141(2014), 1084-1088 (Aug 2014)

9. Weller, M.: Virtual Learning Environments: Using, Choosing and Developing your VLE. Routledge, Abingdon, Oxon, UK (2007) 